\title{
Anabases
}

ANABASES Traditions et réceptions de l'Antiquité

\section{Préhistoire de l'humanité et temps de la cité : l'« Archéologie » de Thucydide}

\section{Pascal Payen}

\section{(2) OpenEdition}

1 Journals

Édition électronique

URL : http://journals.openedition.org/anabases/2765

DOI : 10.4000/anabases.2765

ISSN : 2256-9421

Éditeur

E.R.A.S.M.E.

\section{Édition imprimée}

Date de publication : 1 mars 2006

Pagination : 137-154

ISSN : 1774-4296

\section{Référence électronique}

Pascal Payen, «Préhistoire de l'humanité et temps de la cité : l'« Archéologie » de Thucydide »,

Anabases [En ligne], 3 | 2006, mis en ligne le 01 janvier 2012, consulté le 20 octobre 2019. URL : http:// journals.openedition.org/anabases/2765; DOI : 10.4000/anabases.2765 
Anabases 3 (2006), p. 137-154

\section{Préhistoire de l'humanité et temps de la cité : l'«Archéologie » de Thucydide}

Pascal Payen

\section{Prolégomènes : Thucydide n'est pas un historien}

LES TEXTES qui permettent de comprendre comment les Grecs anciens concevaient la vie de l'homme en société montrent une prévalence presque exclusive du politique, compris comme mode de vie en cité. Dans la Politique, Aristote souligne que "la cité

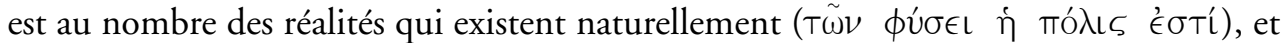

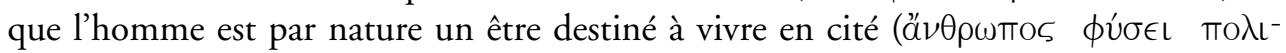

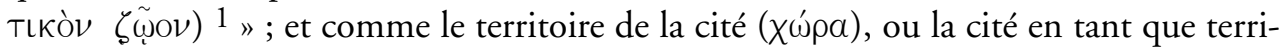
toire, a pour fonction première d'assurer à la communauté politique son « autarcie, en vue de la vie heureuse ${ }^{2}$ ", cela signifie que, par-delà la dimension économique liée à la subsistance du groupe, la cité est à elle-même sa propre fin. Une des conséquences de cette conception du politique appréhendé comme "phénomène social total » est qu’aucun moment du temps, y compris toute reconstitution d'une préhistoire de l'humanité, ne saurait être pensé sans la cité, sans que celle-ci soit déjà advenue, comme si elle était toujours déjà là.

Le premier aboutissement de la réflexion des Grecs sur ces questions est la Politique qu'Aristote écrit, dans sa phase finale, avec l'appui de ses disciples, entre 335 et 323. Pour les temps qui précèdent, c'est Thucydide qui met en œuvre de la manière la plus radicale les modalités et les conséquences d'une omniprésence de la polis, depuis

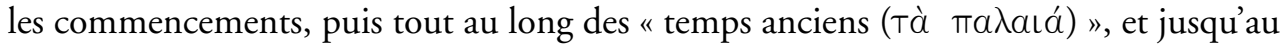
présent $(\nu \tilde{v} \nu)$. Il y procède dans un passage situé au début de La guerre du Péloponnèse

1 Aristote, Politique I, 2, 1253 a 2-3.

2 Ibid., VII, 4, 1326 b 9 ; voir aussi I, 2, 1252 b 28-29 ; VII, 5, 1326 b 28-29. 
- les chapitres 2 à 19 du livre I -, et connu sous le nom d'Archéologie, que lui attribue le scholiaste ${ }^{3}$. Cette description est tantôt celle de l'état présocial ou de la genèse de l'état social de l'homme, tantôt celle des premiers temps des sociétés grecques, alors

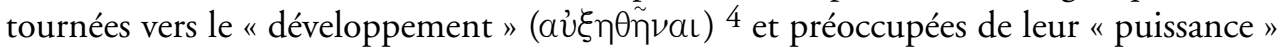

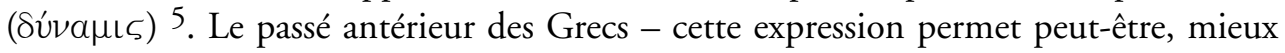
que le terme de "préhistoire ", de marquer la continuité de toute la période comprise avant la guerre du Péloponnèse - doit son unité à deux paradigmes descriptifs. D'une

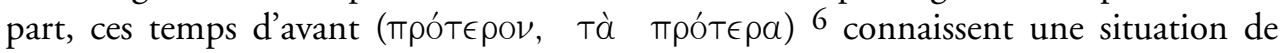
faiblesse $\left(\dot{a} \sigma \theta \dot{\epsilon} v \in\llcorner\alpha)^{7}\right.$ et de manque dans presque tous les domaines, selon un des tableaux traditionnels des origines de la société humaine, tel que le présente par exemple le héros éponyme dans le Prométhée enchaîné d'Eschyle ${ }^{8}$. D’autre part, ils sont tout entiers occupés par une forme d'organisation dénommée polis, sans que celle-ci se confonde toujours, surtout dans la première partie de l'Archéologie, avec la structure institutionnelle et sociale contemporaine de Thucydide. L'étrangeté d'une telle présence continue est renforcée par le fait que les débuts, ceux de la cité comme ceux d'un hypothétique "temps des hommes", ne sont jamais clairement fixés : aucune occurrence du substantif ả $\rho \chi \eta ́$ ou du verbe ă $\rho \chi \in \sigma \theta a \iota$ ne figure dans ces chapitres.

En dépit de ces repères peu conformes à nos attentes d'archéologue et d'historien formés à l'école du positivisme, la particule initiale yáp - "On voit, en effet, que ce qui est appelé maintenant Grèce n'était pas, dans les temps anciens, habitée de façon stable 9 " - indique que tout l'exposé est investi d'une fonction de preuve destinée à confirmer la thèse avancée dès les premières lignes de l'œuvre. Cette thèse tient en deux

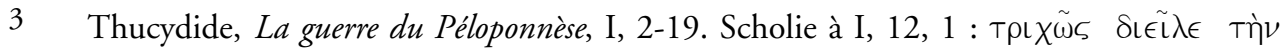

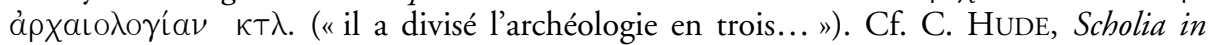
Thucydidem, Leipzig, Teubner, 1863, p. 15. Le texte suivi est celui établi par Jacqueline DE ROMILlY, dans l'édition de la CUF, Paris, Les Belles Lettres, 1953. La traduction prend appui sur celle de Jacqueline de Romilly; elle s'en écarte assez souvent pour mettre en valeur les éléments en rapport avec le sujet de cette contribution. Thucydide I, 2,$6 ; 12,1 ; 16,1$.

Ibid., I, 5, $1 ; 9,1 ; 9,2 ; 10,2 ; 11,1 ; 13,6 ; 18,1 ; 18,2$.

Ibid., I, 2, $1 ; 9,2$.

Ibid., I, 3, $1 ; 3,4 ; 5,1$.

8 Eschyle, Prométhée enchaîné, v. 447-468. Cf. également Platon, Protagoras, 320c-322d, où l'espèce humaine, oubliée par Epiméthée, se trouve dépourvue des qualités nécessaires à sa survie (321c-d), ainsi que Diodore de Sicile, I, 8, qui emprunte à Démocrite la description du "genre de vie le plus ancien (та入аıота́тоu) des hommes" (I, 8, 10). Sur les échos de ces conceptions relatives au progrès, dans l'"Archéologie" de Thucydide, cf. J. DE Romilly, "Thucydide et l'idée de progrès", Annali della scuola normale superiore di Pisa 35 (1966), p. 150-161. La description de la cinquième race, la race de fer, chez Hésiode, Les Travaux et les Jours, v. 174-201, est à lire dans une perspective morale, pour le présent. L'autre tableau attendu est celui de l'âge d'or : par exemple Hésiode, Les Travaux et les Jours, v. 109-125. 
propositions : mon objet d'étude n'est pas une histoire de la Grèce, mais une guerre, " la guerre entre les Péloponnésiens et les Athéniens 10 ", et, seconde proposition, ce

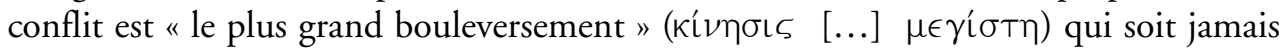
survenu, en comparaison de tous "les précédents 11 ". La description des " temps anciens " se trouve implicitement recouverte du sceau de la "vérité ", dont la

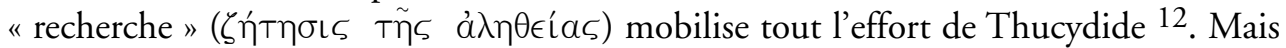
s'il est juste de reconnaître qu'il y déploie tout un ensemble de procédés qui peuvent sonner " en cette fin de ve siècle comme un véritable manifeste " de la raison ${ }^{13}$, y compris par le recours à de complexes compositions annulaires 14, la méthode employée et l'épistémologie qui la sous-tend n'entretiennent que fort peu de rapports avec celles du rationalisme de l'histoire scientifique, telle qu'elle a été élaborée, pour l'essentiel au XIXe siècle, notamment au contact des "sciences de l'Antiquité " (Altertumswissenschaft), en Allemagne et en Europe. Thucydide n'est pas un historien, et il ne fait pas figure de lointain " collègue".

Ce constat résulte d'analyses qui ont permis de préciser, depuis trois ou quatre décennies, les catégories de la démarche thucydidéenne, à partir d'un travail réflexif et critique portant, d'un côté, sur l'histoire des Modernes, en tant que discipline, avec ses

10 Cf. la remarque en ce sens de A. W. Gomme, A Historical Commentary on Thucydides, Oxford, Clarendon Press, vol. I, 1945, p. 89. Sur les liens entre la guerre et la prose historique, depuis Hérodote, on nous permettra de renvoyer à notre étude "L'historien, la guerre, l'écriture, les vaincus (ve siècle avant J.-C. - II siècle après J.-C.)”, Storia della storiografia 41 (2002), p. 47-61.

11 Ibid., I, $1,1$.

12 Ibid., I, 20, 3. C'est pourquoi V. Hunter, Past and Process in Herodotus and Thucydides, Princeton, Princeton University Press, 1982, p. 17, note avec subtilité que Thucydide a certainement quelque chose de très important à faire passer pour se lancer dans une reconstruction si périlleuse, notamment en raison de la rareté et de la difficulté des sources. Sur ce dernier point, qui n'entre pas dans notre analyse, voir le bilan récent de R. NiCOLAI, "Thucydides' Archaeology : Between Epic and Oral Traditions", in N. LuRAGHI ed., The Historian's Craft in the Age of Herodotus, Oxford, Oxford University Press, 2001, p. 267-275 ("Thucydides' Evaluation of his Sources of Information").

13 Cf. J. DE Romilly, Histoire et raison chez Thucydide, Paris, Les Belles Lettres, 1956, p. 244246 (citation, p. 244), 266 et passim. Selon les mêmes présupposés, A.W. Gomme, $A$ Historical Commentary on Thucydides, p. 155-157, réécrit un synopsis de l'exposé de Thucydide avec digressions et notes infra-paginales. Les travaux de C. N. Cochrane, Thucydides and the Science of History, Oxford, 1929, de J.H. FInLEY, Thucydides, Harvard University Press, 1942, 2ème éd. 1947, de K. VON FrITZ, Die grieschiche Geschichtsschreibung, Band I : Von den Anfängen bis Thukydides, Berlin, Walter de Gruyter, 1967, 1. Text, p. 575-617 et 2. Anmerkungen, p. 261-283, voient dans l'Archéologie un puissant effort pour mettre en œuvre les règles de la méthode historique positive, en dépit du manque de sources et de leur difficulté, dans le cas des poètes.

14 Dont la sophistication ne saurait néanmoins être exagérée, comme dans l'étude de J.R. ElLIS, "The Structure and Argument of Thucydides' Archaeology", Classical Antiquity 10 (1991), p. 344-381. 
méthodes, ses courants et ses institutions ${ }^{15}$, et, d'un autre côté, sur un examen des registres lexicaux et des attitudes propres aux premiers prosateurs grecs. C'est un rapport de différence, fort peu d'analogie, que nous entretenons avec eux, même s'il faut noter que de telles analyses sont permises, parce que c'est la notion, ancienne et moderne, d'historia qui en constitue le cadre sur la longue durée. Quelles sont les marques les plus évidentes qui éloignent Thucydide des historiens modernes ?

Tout d'abord, jamais l'historien grec ancien ne se désigne ou n'est désigné par les termes histôr ou historikos. Le premier, au demeurant, a le sens d'" arbitre " ou de "garant ", sollicité pour trancher, par son autorité, ce qu'il n'a pas vu lui-même, sens clairement attesté dans deux scènes de l'Iliade souvent analysées ${ }^{16}$. Le second terme, historikos, est seulement utilisé par Aristote, dans la Poétique ${ }^{17}$, pour opposer au poète le "chroniqueur ", dont il prend Hérodote pour principal représentant, et non Thucydide; le terme ne se généralise pas avant Polybe. Dans la longue durée, depuis Hérodote jusqu’à Lucien, dans son traité Comment il faut écrire l'histoire, ce sont inva-

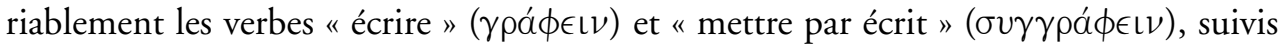
d'un complément d'objet, qui indiquent en quoi consiste l'activité des historiens, et eux-mêmes recourent aux substantifs grapheus ou suggrapheus pour se nommer ${ }^{18}$. Dans le même sens, Thucydide prend soin de ne jamais employer une seule fois historia (ou le verbe $i \sigma \tau о \rho \tilde{\imath} \nu)$, pour se démarquer de l'"enquête " hérodotéenne, et parce que, pour lui, le moyen de connaissance le plus sûr est le constat qu'il opère par la vue ${ }^{19}$; l'objet de toute son œuvre ne pourra donc être qu'un événement contemporain qu'il aura vu lui-même : c'est le principe de l'autopsie, qui interdit toute identification avec

15 Le renouvellement a été sensible depuis les années 1970, lorsque les historiens ont pris pour objet d'analyse les mutations dans les champs, les méthodes et les paradigmes de l'historien. En France, les travaux de Paul Veyne, Michel de Certeau, Paul Ricoeur et François Hartog sont parmi les plus importants.

Iliade XVIII, 501 ; XXIII, 486. Cf. A. SAUGE, De l'épopée à l'histoire. Fondement de la notion d'historiè, Francfort sur le Main, Peter Lang, 1992, p. 103-108 et passim ; E.

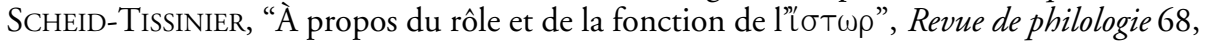
1-2 (1994), p. 187-208 ; F. HARTOG, "Le témoin et l'historien", Gradhiva 27 (2000), p. 67.

17 Aristote, Poétique, 9, 1451 b 1-2.

18 Hérodote I, 95; II, 70, 123 ; IV, 195 ; VII, 214 ; Thucydide I, 1, 1 ; 22, 1 ; II, 1 ; 70, 5 ; 103, 2 ; III, 25, 2 ; 88, 4 ; 116, 3 ; IV, 116, 3 ; 135, 2 ; V, 24, 2 ; 26, 1 ; VI, 7, 4; 93, 4 ; VII, 18, 4 ; Polybe, I, 1, 4 ; I, 2, 8 ; XVI, 14, 6 Denys d'Halicarnasse, Sur Thucydide 5, 1-2 ; Flavius Josèphe, La guerre des Juifs, Préface, 18 ; Antiquités juives I, 1 et 4 ; Arrien, Ananase d'Alexandre I, 1 ; Lucien, Comment il faut écrire l'histoire, 39, 41..., pour s'en tenir à quelques références. 
l'historien moderne. Toutes les autres «traces " : documents écrits ${ }^{20}$, récits de la tradition (ảkoaí) ${ }^{21}$, informateurs, et même son propre avis résultant de ce qu'il n'aurait pas « vu 22 » sont moins fiables. De ce principe fondamental il résulte qu'on ne peut avoir qu'une connaissance imparfaite des temps anciens, puisqu'il n'est possible de les " connaître clairement $(\sigma a \phi \tilde{\omega} \zeta \in \dot{v} \rho \tilde{\iota} \nu)$ " qu’à travers des "indices $(\sigma \eta \mu \epsilon \tilde{\imath} \alpha)$ ) et des

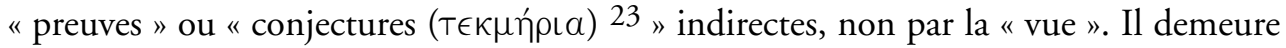
toutefois possible d'explorer le passé. Il existe en effet une nature humaine commune (тò $\alpha \nu \theta \rho \omega ́ \pi เ \nu O \nu)$, vecteur par lequel transmettre la connaissance du présent aux générations à venir, comme un " acquis pour toujours 24 ". C'est en vertu de ce concept central que peut se déployer une exploration du passé, par analogie avec le présent 25. Puisque la nature humaine ne change pas, les critères d'intelligibilité du présent peuvent être déplacés dans les "temps anciens ». On entrevoit que, sur ce point également, ce n'est pas une analyse proprement historique que propose Thucydide, mais une

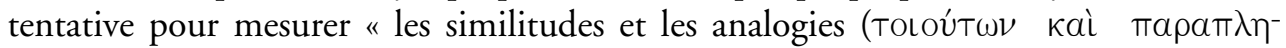
$\sigma i ́ \omega \nu)^{26}$ " de nature structurale qui existent entre présent et passé. Dès lors, il importe peu de fixer précisément un temps des débuts ou une origine. Le passé est convoqué pour montrer à la fois son infériorité par rapport au présent - «j’affirme que les événe-

20 Sur la question des sources écrites de Thucydide - traités, inscriptions, lettres, oracles, serments, décrets - cf. en dernier lieu C. BEARZOT, "L'uso dei documenti in Tucidide", in A.M. Biraschi, P. Desideri, S. Roda e G. ZeCChINI a cura di, L'uso dei documenti nella storiografia antica, Edizioni Scientifiche Italiane, 2003, p. 267-314. Néanmoins, analyser l'attention prêtée aux documents par Thucydide, qu'il est juste de lui reconnaître, ne saurait faire oublier que ces documents ne lui sont pas utiles en tant que "sources", comme chez l'historien moderne, mais qu'ils ont pour fonction principale de conforter le jugement établi au moyen de la "vue ». Le principe de l'" autopsie » est le fondement de l'« histoire véritable».

Ibid., I, 20, 1.

Ibid., I, 22, 2.

23 Ibid., I, 1, 2; 20, 1. Cf. L. CANFORA, “Trovare i fati storici”, Quaderni di storia 13 (1981), p. 212-214. Sur le vocabulaire de la preuve chez Thucydide, dans ses rapports avec l'éloquence du tribunal, cf. P. DESIDERI, "La prova nell'oratoria giudiziaria e nella storiografia nel mondo antico", Quaderni storici 85 (1994), p. 43-57 ; M.-L. DesClOS, Aux marges des dialogues de Platon. Essai d'histoire anthropologique de la philosophie ancienne, Grenoble, Jérôme Millon, 2003, p. 47, 49-62 (avec la bibliographie antérieure), ainsi que C. DARBOPeschansKI, "Thucydide: Historien, Juge", Mètis II, 1 (1987), p. 135-139. Sur le sens le tekmèrion, cf. A. W. Gomme, A Historical Commentary on Thucydides, Volume I, Oxford, Clarendon Press, 1945, p. 135 : "not evidence, but the inference drawn from the evidence". Thucydide I, 22, 4.

25 Ce n'est donc pas l'opposition avec le divin qui est essentiel. Contra W. WYATT, “Thucydides 1.22”, Hyperboreus 2, 1 (1996), p. 191-193. Thucydide I, 22, 4 . 
ments n'y connurent aucune grandeur (oủ $\mu \epsilon \gamma a ́ \lambda \alpha$ ), ni les guerres ni les autres domaines 27 » et la validité définitive des critères forgés à partir de la guerre actuelle.

Ces hypothèses expliquent, du moins pour une part, que l'Archéologie occupe une fonction structurante dans l'œuvre de Thucydide. Ce passage a vraisemblablement été rédigé entre 400 et 396-395. Certes la question n'a cessé d'alimenter l'histoire de l'érudition, mais ces deux dates ont été proposées avec de bons arguments dans deux études de Jean Pouilloux et François Salviat. Le terminus de 396-395 est retenu à partir d'un nouvel examen de la liste des archontes de Thasos pour l'année 398/7, où figure le Spartiate Lichas. Celui-ci, bien connu de Thucydide, est évoqué notamment au livre VIII ${ }^{28}$, au moment de sa mort, qui n'a pu survenir qu'après son archontat, en un temps où Thucydide était engagé dans l'écriture de son ouvre ${ }^{29}$. La date de 400 est établie à partir du passage de l'Archéologie 30 où il est précisé que Sparte exerce son

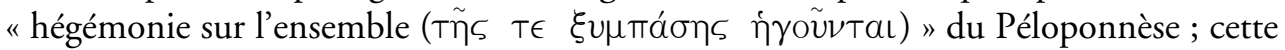
donnée ne peut que renvoyer à une situation postérieure à 400, lorsque la guerre conduite par le roi Agis permet de soumettre les Éléens, en révolte depuis 420, et de rendre aux Lacédémoniens l'accès au sanctuaire d'Olympie ainsi que la maîtrise totale de la région. Composé au moment de la rédaction finale et continue, ce passage n'est donc en aucune manière une "digression 31 ». Il est placé entre l'énoncé du sujet et de la thèse, et les chapitres méthodologiques (I, 20 à 23) ; il se présente donc, d'une part, comme une "préhistoire " de la "guerre entre les Péloponnésiens et les Athéniens ", et il a pour fonction de mettre au jour la genèse de l'état social - la cité - qui rend possible cette guerre ; il se présente, d'autre part, comme une mise à l'épreuve des paradigmes valables pour l'ensemble de la guerre du Péloponnèse, mais aussi comme l'exposé de la découverte de ces mêmes paradigmes tout au long de l'histoire primitive de la Grèce et de la cité.

C'est ce constant double registre qui sera analysé de trois points de vue : en examinant, tout d'abord, comment les " temps anciens " sont désignés et quel rapport au passé antérieur se construit dans la langue ; en étudiant, ensuite, quelles sont les catégories élaborées par Thucydide, à la fois modèles importés et paradigmes sans cesse

Ibid., I, 1, 2.

28 Thucydide VIII, 84, 5. Cf. J. Pouilloux et F. Salviat, "Lichas, Lacédémonien, archonte à Thasos et le livre VIII de Thucydide", Comptes rendus de l'Académie des Inscriptions et Belles Lettres, 1983, p. 376-403. P. CARTLEDGE, "A new lease on life for Lichas son of Arkesilas", Liverpool Classical Monthly 9 (1984), p. 98-102, émet des réserves sur l'identification entre le Lichas de Thucydide et celui de la liste des archontes de Thasos, mais sans apporter de preuve décisive. Cf. J. PouillouX et F. SAlviat, “Thucydide après l'exil et la composition de son Histoire”, Revue de philologie 59, 1 (1985), p. 16-18. Thucydide I, 10, 2.

31 Affirmer que Thucydide, à peine le sujet de l'œuvre formulé, commencerait par une digression va, par ailleurs, à l'encontre de la thèse de l'historien modèle de rigueur ! 
ajustés tout au long de l'Archéologie, pour parvenir à une connaissance neuve des temps d'avant ; en cherchant, enfin, comment situer la préhistoire dans son articulation avec le présent de l'autopsie et avec le futur du ktèma es aiei.

\section{Les « temps anciens": dans le temps et hors du temps.}

Commençons par mettre de côté le problème de la dénomination par arkhaiologia, qui ne provient pas de Thucydide et sur laquelle nous reviendrons.

En 1985, Raymond Weil faisait paraître une étude consacrée à la question des commencements chez les historiens, à partir d'une analyse de la répartition et du sens des adjectifs ápxã̃os et ma入aıós. Il concluait notamment qu'il fallait traduire

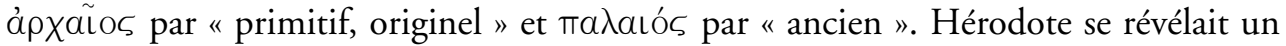
historien "féru d'arkhaion ", toujours en quête des origines et fort peu intéressé par l'aspect ancien des choses ${ }^{32}$. Cette distinction entre «primitif » et " ancien " est aussi celle qu'indique le $D E L G{ }^{33}$. Or ces conclusions et les remarques convergentes d'une brève étude concernant Thucydide et Polybe 34 s'accordent mal avec les chapitres que Thucydide consacre à la préhistoire de la Grèce et du monde barbare. Son analyse remonte à un temps "d'avant " ( $ா \rho O ́ T \epsilon \rho O \nu)$, au-delà de Minos 35 , lorsque l'Attique,

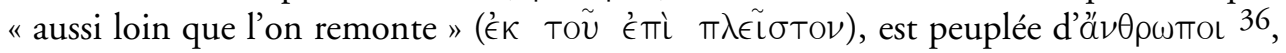
comme dans un temps pré-civique et immobile - ces « hommes " sont " toujours les mêmes" (oí aủtoì al̇é́) -, et pourtant ce n'est jamais l'adjectif arkhaios qui est employé, mais toujours palaios, dont on trouve treize occurrences 37 . Ainsi, « ce que

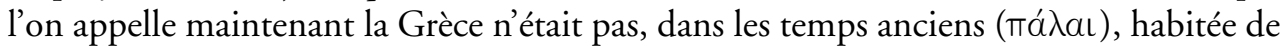
façon stable "; aux temps de Minos, de la guerre de Troie, des tyrans, on vit "à la

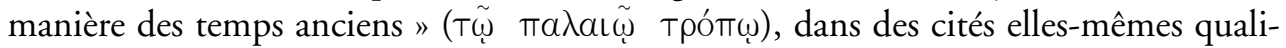

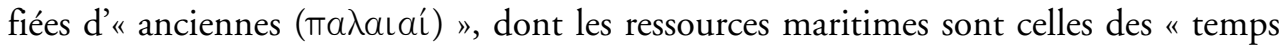
anciens ( Tà Ta入aıá)».

32 Cf. R. WeIL, "Par quoi commencer ?", Storia della storiografia 7 (1985), p. 28-37 (p. $35-$ 36).

33 Cf. P. Chantraine, Dictionnaire étymologique de la langue grecque. Histoire des mots, Paris,

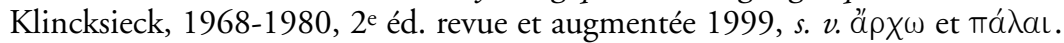

Cf. R. WeIL, "Retour au commencement", Revue de philologie 59, 2 (1985), p. 197-198. Thucydide I, 3-4.

Ibid., I, 2, 5.

Ibid., I, 2,$1 ; 3,1 ; 4 ; 5,1 ; 5,2 ; 5,3 ; 6,5 ; 6,6 ; 7 ; 10,3 ; 13,5 ; 15$. Il est étonnant que S. Hornblower, A Commentary on Thucydides, volume I, Books I-III, Oxford, Clarendon Press, 1991, p. 3, ne relève pas ce fait et fasse au contraire comme si Thucydide ne recourait qu'à arkhaios et était l'auteur du titre attribué par la tradition à ce passage. A. W. Gomme, A Historical Commentary on Thucydides, Volume I, ne commente pas ces termes. 
Dans une étude parue en 2004, Michel Casevitz est revenu sur ce problème. Il note en particulier - l'étude embrasse toutes les données de la littérature grecque - que chez les lexicographes (Hésychius, Pollux, la Souda) les deux termes sont interchangeables, et que chez Hérodote arkhaios a souvent le sens d' " ancien », inscrit dans la durée du passé, et ne marque pas l'origine ${ }^{38}$. Ainsi les Athéniens, lorsqu'ils réclament

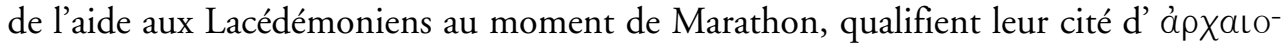

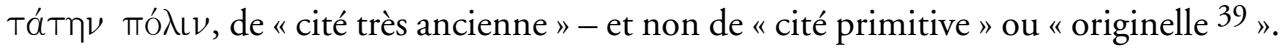
Son étude parvient, d'une part, à la conclusion que palaios et arkhaios peuvent désigner l'un et l'autre l'ancienneté qui remonte à un point de départ et couvrir pareillement toute l'étendue du temps depuis l'origine. Elle montre, d'autre part, qu'une opposition existe bien entre les deux termes ; mais elle tient à leur lien avec la notion grammaticale de personne, au sens où l'entend Benveniste. Arkhaios se rapporte au " je ", au " tu ", à ce qui concerne hic et nunc les locuteurs ; avec arkhaios, le passé affleure le présent et l'explique, comme dans le passage du livre VI d'Hérodote : la cité est qualifiée d'arkhaiotatèn, parce que c'est celle de ceux qui parlent et qui réclament de l'aide pour elle, au nom de son ancienneté, de son passé. Palaios, lui, se rapporte à une chose non impliquée dans la relation avec les locuteurs ; le passé est perçu comme distinct, différent ; "il faut se pencher vers lui, sans qu'il vienne à nous ", note avec finesse Michel Casevitz ${ }^{40}$. Entre les deux mots, se dessinent deux conceptions du passé : avec arkhaios, le passé est de l'ordre du proche, du même, de l'encore existant, qui provient de la valeur subjective de ce mot ; avec palaios, s’impose un passé lointain, différent, révolu.

Lorsque Thucydide fait le choix délibéré de désigner le temps, depuis les origines jusqu'au présent, exclusivement par le terme palaios, il se livre à une opération d'objectivation du passé, transformé en un objet de connaissance, comme dans la phrase qui récapitule, au début du chapitre 20, tout l'exposé en associant ta palaia et le verbe heuriskein : "Tels furent les temps anciens, d'après mes recherches (Tà $\mu \epsilon ̀ v$ oûv

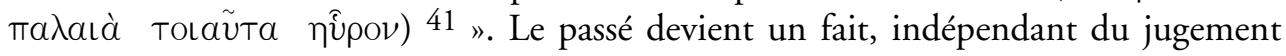
(nous reviendrons sur le sens de heurein). Par là même, dans tous les passages où se

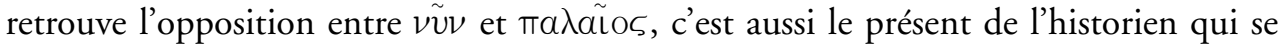

38 Cf. M. Casevitz, "Remarques sur le sens de ảpxãos et de ma入aıós", Métis n. s. 2 (2004), p. 125-136 (p. 126-127). Hérodote VI, 106.

40 Cf. M. CASEVITZ, "Remarques", p. 134-135.

41 Thucydide, I, 20, 1. Les textes concernant les temps premiers dans l'Antiquité sont rassemblés dans le recueil de A. O. LOVEJOY et G. BOAS, Primitivism and related Ideas in Antiquity, with Supplementary Essays, Baltimore, Johns Hopkins Press, 1935 (Paperback ed. 1997), mais, étrangement, Thucydide en est absent, hormis pour quelques brèves références ponctuelles. 
trouve objectivé 42 . La seule occurrence de arkhaios au début de l'œuvre se trouve dans les chapitres de méthode, après l'Archéologie, mais Thucydide n'est plus enfermé dans son analyse ; il rapporte "l'habitude universelle qui veut que, [...] quand une guerre a

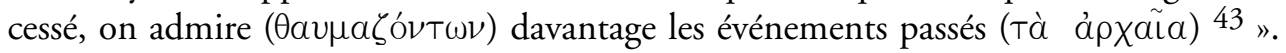
La valeur subjective du verbe thaumazein appelle, autant que les paroles rapportées, le recours à arkhaios pour désigner le passé. Le choix du terme arkhaiologia par le scholiaste pourrait s'expliquer soit par le rapprochement des deux termes, tel qu'on le constate dans les lexiques tardifs, soit par l'influence des désignations rencontrées chez Denys d'Halicarnasse et chez Flavius Josèphe ${ }^{44}$. Il semble que la dérivation de arkhaios à partir de arkhè « commencement » l'ait clairement emporté pour des œuvres qui valorisent explicitement le temps des origines.

Les « temps anciens " constituent ainsi chez Thucydide une durée de vaste étendue, à l'intérieur de laquelle l'exposé distingue un ensemble d' "origines " désignées par le terme $\pi \rho \tilde{\omega} T$ OS. Les compagnons d'Achille sont " les premiers " à porter le nom d'Hellènes ; "Les tout premiers à quitter les armes furent les Athéniens " et les Lacédémoniens à se montrer nus dans les concours ; Pélops «le premier » s'acquit de la

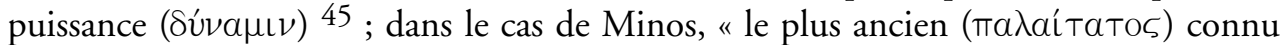
par la tradition " à établir une thalassocratie, la synonymie entre prôtos et le superlatif de palaios montre que le champ sémantique de palaios comporte la notion d'origine 46 . En se fondant sur l'analyse des " manières grecques de commencer " recensées par Marcel Detienne, on constate de nouveau que, chez les Grecs, rien n'est créé ex

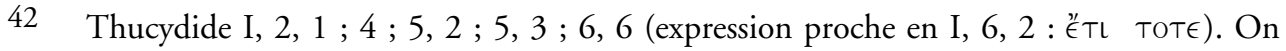
remarquera que le mot *paléologia n’a pas été créé par les Anciens pour désigner une science objective du passé, et le terme arkhaiologia ne s'est pas non plus imposé. Les titres qui nous sont parvenus pour les œuvres de Denys d'Halicarnasse ('A

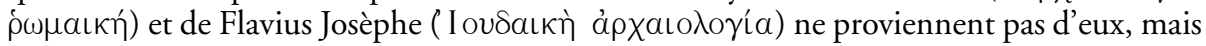
de la tradition manuscrite postérieure, et le terme arkhaiologia désigne à cette époque, comme chez Platon (Hippias Majeur 285d), tout ce qui se rapporte aux périodes anciennes, d'où indifféremment le pluriel (Diodore I, 4, 6;9, 5) ou le singulier (Denys d'Halicarnasse I, 4, 1 ; Flavius Josèphe, Préface 5), sans que les auteurs soient toujours des historiens (Flavius Josèphe, Antiquités juives I, 107-108, range Hésiode aux côtés d'Hécatée, Hellanicos, Acousilaos, Manéthon). On lui a préféré historia, à partir du IIe siècle avant notre ère, peut-être parce qu'il renferme les deux registres sémantiques. Thucydide I, 21, 2. Hérodote est certainement visé, dont le projet est de sauver de l'oubli " les grandes et étonnantes $(\theta \omega \mu \alpha \sigma \tau \alpha ́)$ actions accomplies par les hommes ", notamment les raisons des guerres qui les opposèrent (Prooimion).

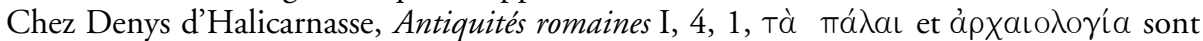
synonymes pour désigner la matière de l'œuvre : comparer à quelques lignes de distance :

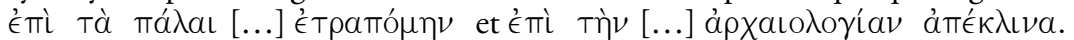
Thucydide I, 3, 3; 6, 3; 9, 2.

Ibid., I, 4. 
nihilo ${ }^{47}$, que le geste de fonder-inaugurer-commencer prend place dans un temps déjà semé de repères ${ }^{48}$. C'est "dès l'époque ancienne " (ảmò ma入aıoũ) qu'Athènes et l'Attique envoyèrent des "colonies " (ảmotкías) en Ionie ; les fondations de cités

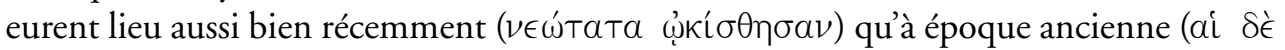
Ta入aıaí) ${ }^{49}$. La prehistoire s'inscrit ainsi dans la sphère du déjà ; la genèse de l'état social ne constitue pas une époque séparée. Comme si l'effort d'objectivation du passé avait unifié chronologiquement les origines, la préhistoire et les lisières du temps présent. Mais raisonner ainsi est en partie impropre, car c'est sur un autre plan, celui de la mise en place des paradigmes de l'analyse, que se construit le rapport entre « temps anciens " et "présent ".

Avant d'aborder la deuxième étape de l'analyse, deux précisions sont nécessaires. Tout d'abord, l'Archéologie n'est pas dépourvue d'indications chronologiques, sous deux formes. L'exposé est ordonné autour de successions qu'il faut bien qualifier d'abord de chronologiques, parce qu'elles correspondent à l'ordre réel ou traditionnel des événements : successions des "puissances " dans le monde grec, avec Minos, Agamemnon, les Tyrannies, Athènes et Sparte ; succession des guerres qui méritent d'être évaluées en terme de "grandeur ": guerre de Troie, deuxième guerre Médique, guerre du Péloponnèse. Par ailleurs, Thucydide dispose dans son analyse tout un ensemble de repères qui permettent d'établir ou de conforter une chronologie relative des faits : le Corinthien Ameinoclès construisit des trières pour Samos « trois cents ans avant la fin de notre guerre "; le plus ancien combat naval, entre Corinthiens et Corcyréens, eut lieu " deux cent soixante ans avant la même date 50 "; " c'est peu avant la mort de Darius " que se généralise l'usage de la trière ${ }^{51}$; la guerre de Troie sert de repère pour situer de nombreux faits " avant " ou "après " elle ${ }^{52}$. Néanmoins, nous le verrons, ce cadre chronologique, à la fois nécessaire et presque formulaire dans la prose historique, ne constitue pas la trame principale qui assure la cohérence de l'ensemble.

Une seconde précision est nécessaire. La Guerre du Péloponnèse comporte deux autres passages qui pourraient être qualifiés d'Archéologie 53. Lorsqu'à l'automne 431, les Athéniens acceptent la stratégie insulaire de Périclès et, depuis les campagnes, se

47 Cf. M. Detienne, "Manières grecques de commencer", in Id. dir., Transcrire les mythologies. Tradition, écriture, historicité, Paris, Albin Michel, 1994, p. 159-160.

Cf. A. Kleingünther, Prôtos Heuretès. Untersuchungen zur Geschichte einer Fragestellung, Leipzig, Philologus Suppl. B, 26-1, 1933, ainsi que M. CASEVITZ, Le vocabulaire de la colonisation en grec ancien. Étude lexicologique : les familles de $\kappa \tau i \zeta \zeta \omega$ et de oiké $\omega-$ oikí $\zeta \omega$, Paris, Klincksieck, 1985.

Thucydide I, 7.

50 Ibid., I, 13, 3-4.

51 Ibid., I, 14, 2.

52 Ibid., I, 12, $1 ; 12,3$.

53 C'est ainsi qu'ils sont rassemblés dans le livre de J. M. AlOnso NúÑEZ, Die Archäologien des Thukydides, Konstanz (Xenia, 45), dont l'analyse n'apporte pas d'élément neuf. 
replient "dans la ville 54 ", Thucydide explique que le même processus s'est produit au moment du synoecisme de Thésée. Les Athéniens, ayant vécu jusque-là " par petites cités séparées ", durent se réunir " en une unique cité 55 ". Cet épisode est situé dans

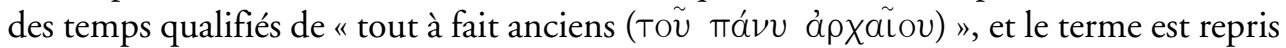
chaque fois qu'une dimension affective est associée à la description 56. Toutefois, lorsque l'énoncé devient presque purement descriptif 57 ou bien est une intervention du narrateur, d'un « il " à la troisième personne, c'est ta入ã̃os qui est employé 58 . Les deux dimensions du passé sont en rapport constant avec le présent ( $\nu \tilde{v} \nu)$, marqué par la figure de Thésée sous les traits de Périclès, c’est-à-dire doté lui aussi de la " puissance "

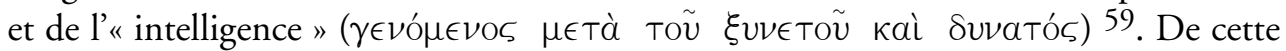
" archéologie attique », on peut rapprocher l' " archéologie sicilienne » du début du livre VI, qui dresse une histoire de l'occupation de l'île, d'abord par les Barbares, puis par les Grecs ${ }^{60}$. Dans ce cas, la présence conjointe de arkhaios et de palaios s'explique certainement par le fait que Thucydide se situe délibérément dans la tradition hérodotéenne ; tout paraît y renvoyer : le retour en arrière sur l'histoire d'un peuple, à peine annoncée une expédition militaire dirigée contre lui ; la description ordonnée en termes d'espace, de territoire (khôra), de peuple (ethnos) et de coutumes, d'usages (nomoi) ; l'énumération qui crée un effet de liste renvoyant, dans la poétique des genres narratifs, au catalogue des vaisseaux de l' Iliade. L'indéniable valeur étiologique à fonction explicative que revêtent ces deux " archéologies " - qui rendent compte de l'unité actuelle de la cité athénienne et de la puissance présente de la Sicile - les distingue de celle du livre I.

La constitution des " temps anciens " en objet d'étude passe donc par deux opérations. Elle résulte avant tout d'un travail de nomination, propre à la pensée grecque. Conjointement la "préhistoire " est soumise au problème de la temporalité : elle est située aussi bien dans le temps, puisque la cité l'emplit tout entier, que hors du temps, car la polis est présente comme en attente de ses caractères propres. Ce dernier point conduit à émettre l'hypothèse que la constitution des " temps anciens " repose sur un ensemble de catégories dont la mise en place et la dimension principale sont moins chronologiques et temporelles que logiques.

Thucydide II, 17, 1.

Ibid., II, 15, 1-2.

Ibid., II, 16, 2 : « Ils trouvaient pénible et supportaient mal d'avoir à quitter des maisons et des sanctuaires qui avaient toujours été les leurs de père en fils. "

Ibid., II, 15, 2.

Ce que relève bien R. WeIL, "Retour au commencement”, p. 198 : «Thucydide ne prend pas parti quant à l'état originel des choses."

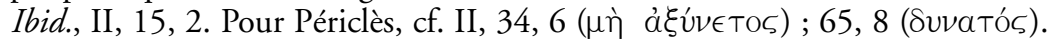

Ibid., VI, 1-2 (Barbares) ; 3-5 (Grecs). 


\section{Thucydide et " l'histoire conceptualisante "}

Le principe cardinal de l'universalité de la nature humaine - kata to anthrôpinon -, formulé dans le chapitre 22, est une déduction des analyses de l'Archéologie. L'« acquis

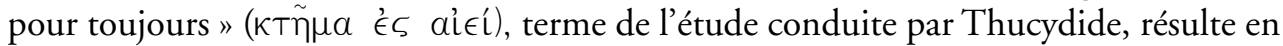

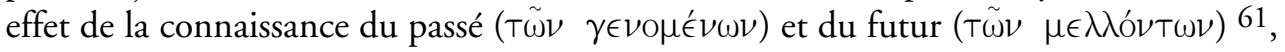
réunis en un " toujours " qui n'est pas un coup d'arrêt porté au mouvement de l'histoire, mais qui souligne ce qu'apporte de définitif la compréhension - la "découverte " -, désormais définie comme un "acquis ", du conflit entre Péloponnésiens et Athéniens. L'énoncé de cette catégorie - l'universalité de la nature humaine - à cet endroit, suggère que Thucydide soumet toute sa réflexion sur les " temps anciens » des sociétés humaines à ce que Lévi-Strauss appelle " le problème de l'invariance 62 ». En effet, pour éviter de conclure, à partir d'un tel principe, que rien n'a jamais changé, il faut faire jouer d'autres catégories, inventer des " concepts " qui permettront d'observer l'objet en question - la guerre du Péloponnèse et les autres conflits - en termes de différence. Le concept englobant que propose Thucydide pour parvenir à une nouvelle

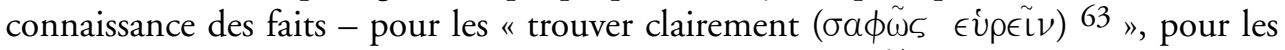
dégager en tant qu'objet d'analyse - est celui de " grandeur 64 »; il permet de placer les données sur une échelle d'évaluation qui fait passer à l'arrière plan la dimension chronologique des faits et la notion d'évolution. Poser une telle notion implique que, pour dégager les différences entre les événements, il faille procéder par comparaison ou analogie, principes formulés conjointement avec celui de l'universalité de la nature humaine. Thucydide se livre ainsi à un travail de création conceptuelle, qui est certainement une réponse à la "découverte de la complication du monde 65 »; ces catégories de l'analyse sont, pour les plus englobantes, déduites de l'Archéologie dans les chapitres dits de méthode qui suivent, et pour les autres, construites au fil l'exposé, comme nous allons le voir, mais aussi, conjointement, importées du présent athénien.

C'est là, en effet, la première conséquence du principe analogique : les sociétés grecques et barbares primitives ne sont pas fondamentalement différentes de celles de " maintenant "; les mêmes catégories peuvent leur être appliquées pour parvenir à les

61 Ibid., I, 22, 4.

62 C. LÉVI-STRAUSS, "Le champ de l'anthropologie", Leçon inaugurale de la chaire d'anthropologie sociale, Collège de France, 5 janvier 1960, Anthropologie structurale deux, Paris, Plon, 1973, p. 35, ainsi que P. Veyne, Linventaire des différences, Paris, Seuil, 1976, p. 22.

63 Thucydide I, 1, 2.

64 Cf. L. CANFORA, "La préface de Thucydide et le critique de la raison historique", Revue des Études Grecques 90 (1977), p. 455-461.

65 Cf. P. Veyne, Comment on écrit l'histoire, Paris, Seuil, 1971, p. 255 (et tout le chap. 10 : "L'allongement du questionnaire"). 
" trouver ", à les connaître. De sorte que si périodisation il y a, celle-ci résultera de l'application de concepts, non de la succession chronologique ou de l'observation des restes actuels, au sens moderne de traces archéologiques. Ainsi est-ce en déplaçant l'analyse en

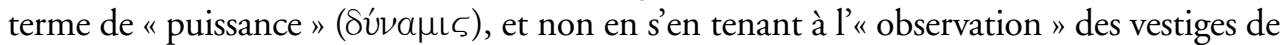
Mycènes - et cela vaut pour n'importe quelle autre cité : Sparte ou Athènes, ajoute Thucydide, jamais en reste de généralisation dans ces pages -, que l'on peut conclure, à propos de la guerre de Troie, que « cette expédition fut plus importante $(\mu \in \gamma(\sigma T \eta \nu)$ que les précédentes, mais inférieure à celles de nos jours 66 \%. Le principe structural l'emporte toujours sur la logique chronologique. Ce sont la «puissance » et les « ressources finan-

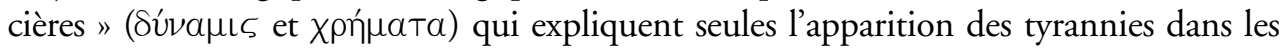
cités, après la guerre de Troie, et non des raisons d'ordre social, qui n'entrent pas dans la liste des concepts de Thucydide. Le binôme palai/nun, présent à six reprises, nous l'avons noté ${ }^{67}$, apparaît moins comme une opposition d'ordre chronologique, que comme une unité conceptuelle qui permet d'appréhender les événements de l'Archéologie à l'intérieur d'un espace unitaire. De là découlent les formules en forme de théorème, qui écrasent et effacent la chronologie : "Jusqu'à notre époque, une grande partie de la Grèce vit à la

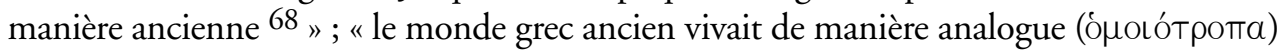

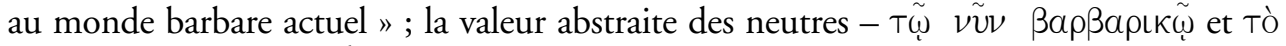

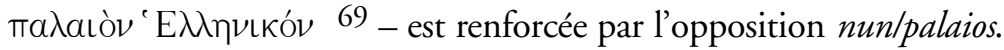

Quels sont ces concepts et comment s'opère le travail de création, au fil des mots et de l'exposé ?

Tout commence, dans la préhistoire du pays grec, par une phase d'instabilité, due aux migrations, et de manque 70 : pas de commerce, pas de "relations sûres", pas de réserves d'argent, pas de plantations, pas de remparts ; et pourtant, les poleis sont déjà

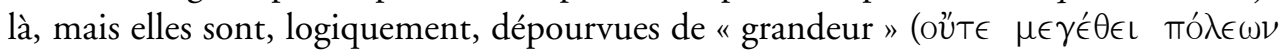

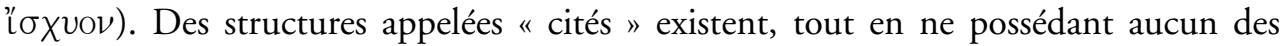
caractères d'une polis. D'emblée, Thucydide dispose les éléments conceptuels dont il a besoin pour la suite, sans réelle préoccupation de "vraisemblance historique ", car l'analyse des temps anciens ne repose pas sur des catégories intrinsèques. La cité, avant la cité, est là comme une forme sinon vide, du moins en attente. L'état social et politique est une donnée en soi, quel que soit le moment des " temps anciens " où l'on se place ; dans le chapitre 2, aucune indication ne permet de relier la description à un contexte historique ou légendaire. D'où ce paradoxe : l'état social et sa genèse ne peuvent être pensés sans la polis, mais il est permis de parler néanmoins d'une préhistoire de la polis, dont les traits, qu'on vient de rappeler, sont définis comme le négatif

66 Thucydide I, 10, 3.

67 Cf. supra, note 42.

68 Thucydide I, 5, 3 ; même formulation en I, 6, 2.

69 Ibid., I, 6, 6.

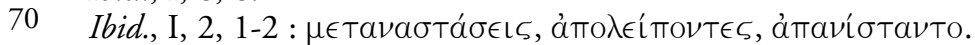


des caractères de l'Athènes de "maintenant ». Paradoxe encore visible à ceci : " depuis

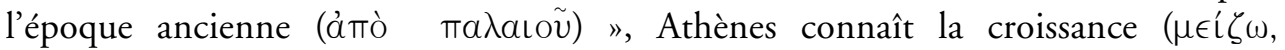

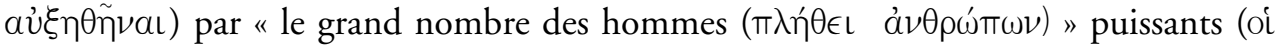

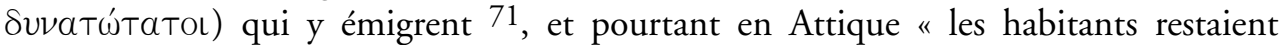
toujours les mêmes (oi aùToì ali $\in \hat{l}$ ) 72 " et l'on y cherche un refuge "stable 73 ". Athènes possède à la fois les traits d'une cité " chaude ", engagée dans les conflits de l'histoire, et ceux d'une cité " froide ", comme si sa mémoire était constituée une fois pour toutes, sous les traits inchangés de ses occupants. Dans le chapitre 3, c'est bien la même démarche qui est à l'œuvre. Alors que les étapes qui ont conduit à la généralisation du nom d'Hellas, dont la connotation est beaucoup plus ethnographique que géographique ${ }^{74}$, et à celui d'Hellènes, sont remplies d'incertitudes, la structure de la polis traverse les phases de la légende - Hellen est fils de Deucalion - et l'époque de la guerre de Troie, jusqu'au temps d'Homère, "lui qui vécut à une époque encore bien postérieure 75 ": les fils d'Hellen, parce qu'ils deviennent puissants, sont réclamés " dans les autres cités ", et par la suite, leur nom se répand "d'abord cité par cité (КaTà

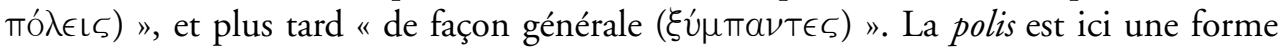
qui, appliquée à tel ensemble de données : le mythe, la guerre de Troie..., permet de comprendre quelque chose au rapport entre temps anciens et présent, ainsi qu'à l'émergence d'une polis du type de celle d'Athènes, qui est un hapax pour Thucydide, car elle est à la fois la source des concepts qui s'élaborent peu à peu dans le passé et l'origine du ktèma es aiei, ce modèle définitif d'analyse qui doit permettre de " comprendre " (heurein) tous les présents à venir.

Prenons un dernier exemple de ce que l'on se risquera à appeler une " histoire conceptualisante 76 " : l'exemple d'une entité constitué de plusieurs éléments. Dans les fondations récentes ( $\nu \in \omega \dot{T} \alpha T \alpha)$ ), les cités se développent grâce à la "navigation "; celleci résulte des " réserves d'argent "; et cette acquisition permet la construction de "fortifications 77 ». La flotte, l'argent, les murs : le triptyque qui constitue le socle de la politique et de la puissance athéniennes après les guerres Médiques est donc projeté sur un temps antérieur ${ }^{78}$. Mais les trois éléments ont acquis force de concept l'un après

Ibid., I, 2, 6.

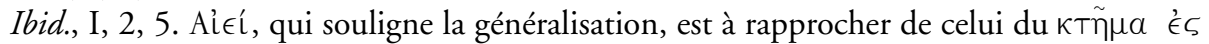
$a \grave{i} \in \mathbf{i}$, qu'il annonce.

Ibid., I, 2, 6.

Ainsi que le notent J. Classen et J. Steup, dans leur commentaire : Thukydides, Vol. I, Berlin, Weidmann, 1900, 5e éd. 1919, p. 7 (I, 2, 1), et 13-14 (I, 3, 1).

Thucydide, I, 3, 1-4.

Cf. P. VeYne, Comment on écrit l'histoire, 145-175, 263-275.

Ibid., I, 7, 1.

Celui de cités telles que Milet, Thasos, Samos, Syracuse, selon A. W. Gomme, Historical Commentary, ad. loc. Mais Thucydide ne donne aucun nom, pour conserver à l'énoncé du processus sa valeur générale. 
l'autre, dans l'exposé qui précède immédiatement. Minos (chapitre 4) est le premier à

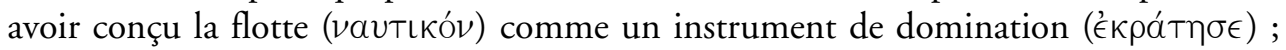
puis, l'exercice de la piraterie sur mer et du pillage sur terre, activités hautement valorisées, ont dégagé des richesses (chapitre 5); celles-ci, enfin, permettent de construire des remparts, donc de profiter de ce sentiment de sécurité pour abandonner les armes (chapitre 6). Dans le sens inverse du mouvement précédent, donc du passé vers le présent, c'est le passé qui, cette fois, prépare la formation du lien organique entre les trois termes. Le processus que nous nous sommes risqué à appeler de création conceptuelle opère donc dans les deux sens du temps; il quadrille le temps.

Tentons d'en prendre une vue d'ensemble, à partir d'une liste des concepts à un terme, et des nœuds conceptuels, à deux ou à trois éléments, créés par Thucydide pour rendre compte de la genèse de l'état social, cette sorte d'impensé de la polis, qu'il s'efforce précisément, dans l'Archéologie, de formaliser.

À partir du postulat de l'universalité de la nature humaine ( $\tau \dot{\alpha} \quad \alpha \dot{\nu} \theta \rho \omega \dot{\pi} \pi \nu 0 \nu$ ), la comparaison et l'analogie permettent de mesurer, sur l'échelle de la grandeur (Tò

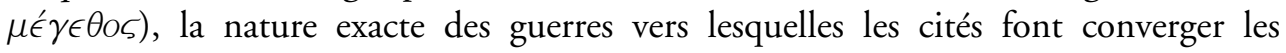

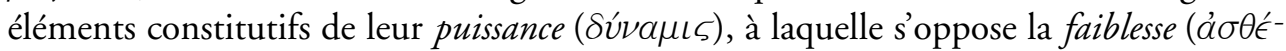
$\nu \epsilon \iota \alpha)$. La puissance se mesure à la solidité de l'articulation entre navires, réserves financières et remparts. L'espace où se déploie l'analyse est constitué d'un temps dominé par la forme de la cité (Tódıs) et d'une préhistoire, d'un temps antérieur, où les hommes

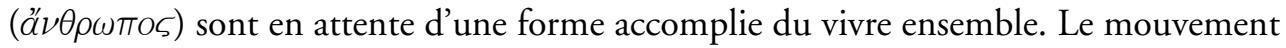
d'ensemble parait suivre la forme d'une progression linéaire, inscrite dans une chronologie, avec ses avants et ses après, ses évolutions, ses ruptures. Mais cette structure de surface est déterminée par un ensemble de concepts, opératoires en fait à toutes les

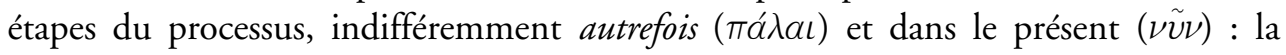
"préhistoire de l'humanité " est présente à tout moment, de même que les virtualités du « temps de la cité ». De sorte que l'opposition fondamentale est peut-être celle entre " humanité » et " cité ", entre ä $\nu \theta \rho \omega \pi$ ol et Tó $\lambda \iota s$, d'une part, parce que l'une et l'autre entité sont présentes à chaque scansion ou époque, d'autre part, parce que les anthrôpoi, les hommes génériques, deviennent des andres, des hommes citoyens, si et seulement si une polis constitue le cadre disponible pour une telle transformation. Thucydide charge Nicias de formuler cet axiome dans les paroles que prononce le stra-

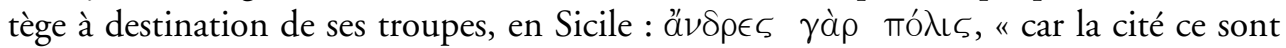
les citoyens ", «les citoyens sont la cité 79 ».

Pour Thucydide, les transformations des sociétés sont de même nature dans les temps anciens et dans le présent. L'ordre diachronique est subordonné à une logique synchronique, et le primitivisme, moment du fonder et de l'inaugurer, du « premier inventeur " (prôtos heuretès), n’est ni réfugié dans un temps lointain, ni isolé du présent ;

Ibid., VII, 77, 7. 
il concentre des événements réunis et mémorisés dans une totalité synchronique et non dans une série diachronique.

Les choix de Thucydide dans la manière de raconter la préhistoire semblent ainsi conduire à une apologie du présent, ou du moins invitent à une clarification des articulations entre passé, présent et futur.

\section{La préhistoire racontée : une apologie du présent ?}

Les différents états de civilisation, que nous étions enclins à considérer comme se succédant dans le temps, du mythe à la cité - selon des catégories dont la validité mérite bien d'être mise sur le métier 80 -, de Deucalion et Minos à Sparte et Athènes, correspondent tout autant aux différentes pièces d'un raisonnement qu'il est toujours possible d'appliquer dans un espace donné ou, si l'on préfere, à chaque instant du temps ${ }^{81}$. Dès lors, si l'on peut parler de "progrès 82 " pour rendre compte, dans l'Archéologie, du passage d'une étape à une autre, c'est en vertu de l'application aux " temps anciens » des idéaux et de l'histoire des idées contemporains de Thucydide (ce qui est certes une dimension du texte et de la pensée de son auteur), mais non en raison d'un processus historique, d'une conception de l'histoire comme processus, avec ses lois, sa progression inexorable. La preuve en est qu'après le conflit entre Athènes et Sparte, il n'y aura pas

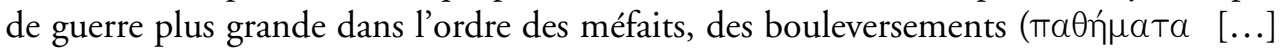
oía oủx Ë $T \in \rho a)^{83}$. Là s'arrête le progrès auquel il est possible de parvenir en terme de connaissance. L'on peut donc dire, avec Lévi-Strauss, que « c'est seulement de temps à autre que l'histoire est cumulative, c'est-à-dire que les comptes s'additionnent pour former une combinaison favorable 84 ». Ainsi y eut-il progrès pour l'Athènes du temps de Périclès : c'est alors " qu'elle fut la plus grande ( $\left.\mu \epsilon \gamma^{\prime} \sigma \tau T\right)$; et de même, lorsque

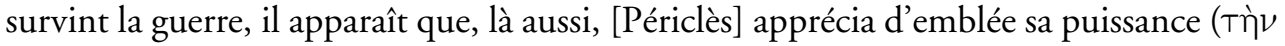
Súvaulv) 85 \%. Mais, contrairement à la thèse la plus répandue chez les historiens modernes, Thucydide souligne que, durant tout le temps des tyrans, « il ne s'accomplit

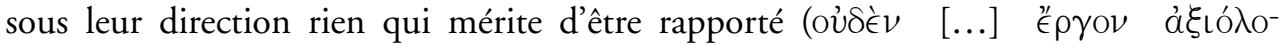
yov) 86 ", précisément parce que, si c'est la guerre qui permet de situer un état historique sur une échelle de valeur, si c'est le principe qui domine la grille d'analyse, alors

80 Cf. M. Detienne, Linvention de la mythologie, Paris, Gallimard, 1981, p. 9-49, 225-242, et C. Calame, Mythe et histoire dans l'Antiquité grecque, Lausanne, Payot, 1996, p. 9-46.

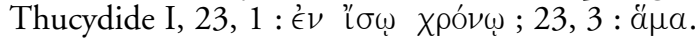
Cf. J. DE Romillly, "Thucydide et l'idée de progrès". Thucydide I, 23, 1. Cf. C. LÉvi-STrauss, Race et histoire, Unesco, 1952, repris dans Anthropologie structurale deux, Paris, Plon, 1973, p. 394. Thucydide, II, 65, 5 . Ibid., I, 17. 
les tyrans sont hors du coup ; la puissance militaire ne fut pas, sauf exception, la marque distinctive de ces régimes 87.

Le récit sur le passé antérieur possède donc fort peu une fonction chronologique, qui expliquerait le présent par ce qui le précède, avec l'idée que plus on remonte haut dans le temps, plus la fonction explicative irait croissant. Ce récit s'inscrit bien plutôt dans le programme de "recherche de la vérité 88 ". Les contenus de tradition que Thucydide consigne dans l'Archéologie comportent une proposition de vérité qui, quel que soit leur teneur historique et le jugement de valeur que l'on peut porter sur eux, concernant par exemple la piraterie, les mœurs, la politique des tyrans, le degré de " grandeur " de la guerre de Troie, contribuent à " voir clair " aussi bien dans " le passé "

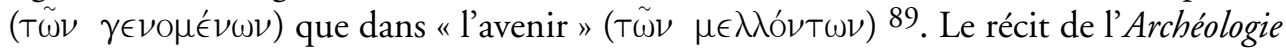
montre que la distance temporelle qui nous sépare du passé est porteuse de sens; les " temps anciens " sont les prémisses (et moins les prémices) qui permettent de mettre en place un échange entre passé et présent, passé et futur. Dans ce processus, est-ce le présent qui sert de repère et de point de jonction ? Quelle est la nature des articulations entre " temps anciens ", présent et futur?

Trois éléments d'analyse peuvent être proposés. Tout d'abord, les « temps anciens " ne sont pas séparés du présent ; on peut parvenir à une connaissance de ce qu'ils sont au moins à partir d' "indices ". Toutefois, ce lien entre passé et présent ne conduit pas à considérer le passé comme un réservoir d'exempla, de modèles, qui feraient que le présent et l'avenir tendraient à répéter le passé. Avec Thucydide, nous ne sommes pas à l'intérieur de ce que François Hartog nomme « le régime ancien d'historicité ", résumé par les mots de Cicéron, dans le De Oratore, où l'histoire, " messagère du passé (nuntia vetustatis) ", est dite " maîtresse de vie (magistra vitae) 90 ». Deuxième élément d'analyse, qui découle du précédent : bien que le passé ne soit pas coupé du présent, il est néanmoins constitué en objet d'étude, mais non selon les méthodes et l'épistémologie de la science historique du XIXe siècle. Cela tient à ce que la rigueur et l'exactitude thucydidéennes 91 ne s'exercent pas sur des documents et des traces issus directement du passé, mais sur des indices inférés à partir d'analogies avec le présent. Enfin, ce n'est pas non plus le futur qui puisse être le vecteur d'une interprétation du passé ni qui éclaire l'histoire, puisque le futur n'existe, au plan de la connaissance, que sous la forme de présents à venir, appréhendés à partir de l'analyse du seul présent qui compte, celui de " la guerre des Péloponnésiens et des Athéniens ", constitué en "acquis pour toujours ». Il n'est nullement question, avec Thucydide, de prévoir l'avenir - l'his-

87 Thucydide ajoute d'ailleurs que seules méritent d'être retenues « des actions les opposant à leurs voisins respectifs " (I, 17).

Ibid., I, 20, 3.

89 Ibid., I, 22, 4 .

90 Cicéron, De Oratore II, 36. Cf. F. HARTOG, Régimes d'historicité. Présentisme et expériences du temps, Paris, Seuil, 2003, p. 84-89 et passim. 
toire n'est pas un processus -, mais de fournir un outil de déchiffrement pour les présents à venir. Thucydide ne s'adresse aux hommes de l'avenir, pour leur être " utile 92 ", que dans la mesure où ceux-ci partagent avec ceux du présent une commune nature humaine. De même peut-on remonter dans les "temps anciens ", en faisant de chaque moment un présent analogue à tous les autres et susceptible, par conséquent, de recevoir la même grille d'analyse. Pour comprendre le passé tel que l'a raconté Thucydide dans l'Archéologie, le détour par le présent est nécessaire, non que ce dernier soit l'unique référence pour comprendre, mais parce que les "temps anciens", auxquels on ne peut accéder en raison de "l'éloignement du temps ", sont aussi le lieu où s'exerce avec une égale force une fermentation conceptuelle. La préhistoire est un présent parmi d'autres, de même que le présent se retrouve dans la préhistoire.

La préhistoire de Thucydide, son Archéologie, n'est donc nullement " un argumentaire idéologique au service du présent 93 "; elle n'est pas non plus une phase première d'un développement historique ; elle se présente encore moins comme une altérité indéchiffrable.

Elle entretient avec le présent une relation de distance et d'interférence. De distance, parce que le recours au terme ta palaia pour désigner ce moment marque une intention d'objectivation ; d'interférence, parce que les modèles d'intelligibilité sont, par leur origine, empruntés au présent. Mais Thucydide construit une fiction logique et chronologique par laquelle il montre que les concepts se sont forgés, sédimentés, complétés, complexifiés au cours des " temps anciens ". Qu'il s'agisse bien d'une démarche conceptuelle, non d'un exposé dont la logique emprunterait seulement le fil de la chronologie, est attesté par la présence de la polis, d'un bout à l'autre, moins un idéal-type wébérien qu'un invariant, nécessaire pour parvenir à une « connaissance » au moins approximative des temps anciens.

L'Archéologie décrit à la fois les temps anciens et le présent, le présent dans les temps anciens; elle recourt à des concepts tirés du présent, mais validés parce qu'ils sont opératoires dans les temps des origines. Ces derniers ont ainsi une fonction légitimante pour l'ensemble de La guerre du Péloponnèse.

\begin{tabular}{l} 
PASCAL PAYEN \\
\hline Université de Toulouse II-Le Mirail \\
U.F.R. d'Histoire, Arts et Archéologie \\
5, allées Antonio Machado \\
31058 Toulouse Cedex 9 \\
payen@univ-tlse2.fr
\end{tabular}

92 Thucydide I, 22, 4.

93 Impasse signalée par P. Cordier dans la présentation de la Journée d'étude, organisée le 25 mars 2005. Cf. sa présentation supra. 